\title{
The association between thrombocytosis and subtype of lung cancer: a systematic review and meta-analysis
}

\author{
Melissa Barlow $^{1}$, Willie Hamilton ${ }^{1} \wedge$, Obioha C. Ukoumunne ${ }^{2} \wedge$, Sarah E. R. Bailey ${ }^{1} \wedge$ \\ ${ }^{1}$ University of Exeter Medical School, St Luke's Campus, Exeter, UK; ${ }^{2}$ NIHR ARC, SW Peninsula, University of Exeter Medical School, St Luke's \\ Campus, Exeter, UK \\ Contributions: (I) Conception and design: M Barlow, SER Bailey, W Hamilton; (II) Administrative support: SER Bailey, W Hamilton; (III) Provision \\ of study materials or patients: None; (IV) Collection and assembly of data: M Barlow, SER Bailey; (V) Data analysis and interpretation: M Barlow, \\ OC Ukoumunne; (VI) Manuscript writing: All authors; (VII) Final approval of manuscript: All authors. \\ Correspondence to: Dr. Sarah E. R. Bailey. College House, University of Exeter Medical School, St Luke’s Campus, Heavitree Road, Exeter, EX1 2LU, \\ UK. Email: S.E.R.Bailey@exeter.ac.uk.
}

Background: Thrombocytosis is associated with poor lung cancer prognosis and has recently been identified as having a high positive predictive value in lung cancer detection. Lung cancer has multiple histological and genetic subtypes and it is not known whether platelet levels differ across these subtypes, or whether thrombocytosis is predictive of a particular subtype.

Methods: PubMed and Embase were systematically searched for studies that reported pre-treatment platelet count, as either averages or proportion of patients with thrombocytosis, by subtype of lung cancer using a prespecified search strategy. The Newcastle-Ottowa scale was used to assess study quality and risk of bias. Suitable studies were synthesised in meta-analyses and subgroup analyses examined for differences across subtypes.

Results: The prevalence of pre-treatment thrombocytosis across all lung cancer patients was $27 \%$ (95\% CI: $17 \%$ to $37 \%$ ). By subtype, this was $22 \%$ (95\% CI: $7 \%$ to $41 \%$ ) for adenocarcinoma, $28 \%$ (95\% CI: $15 \%$ to $43 \%$ ) for squamous cell carcinoma (SCC), 36\% (95\% CI: 13\% to 62\%) for large cell carcinoma (LCC), and $30 \%$ (95\% CI: $8 \%$ to $58 \%$ ) for small cell lung cancer (SCLC). The pooled mean platelet count for lung cancer patients was $289 \times 10^{9} / \mathrm{L}$ (95\% CI: 268 to 311 ). By subtype, this was $282 \times 10^{9} / \mathrm{L}$ (95\% CI: 259 to 306 ) for adenocarcinoma, $297 \times 10^{9} / \mathrm{L}$ (95\% CI: 238 to 356) for SCC, $290 \times 10^{9} / \mathrm{L}$ (95\% CI: 176 to 404$)$ for LCC, and $293 \times 10^{9} / \mathrm{L}$ (95\% CI: 244 to 342 ) for SCLC. There was no difference in thrombocytosis prevalence $(\mathrm{P}=0.76)$ or mean platelet count $(\mathrm{P}=0.96)$ across the subtypes.

Conclusions: These findings suggest thrombocytosis is no more indicative of one lung cancer subtype over another. We therefore conclude a high platelet count is likely to be generic across all lung cancer subtypes.

Keywords: Lung cancer; thrombocytosis; platelets

Submitted Nov 18, 2020. Accepted for publication Dec 17, 2020.

doi: $10.21037 /$ tcr-20-3287

View this article at: http://dx.doi.org/10.21037/tcr-20-3287

\section{Introduction}

Lung cancer is the leading cause of cancer death worldwide. Patients often present at an advanced stage and as a result, the 5-year survival rate for lung cancer in the UK is only $16.2 \%$ (1). As such, research has focused on identifying biomarkers that can help identify lung cancer at earlier stages and therefore improve survival rates. Thrombocytosis

\footnotetext{
^ ORCID: Melissa Barlow, 0000-0002-0746-1868; Willie Hamilton, 0000-0003-1611-1373; Obioha C. Ukoumunne, 0000-0002-05519157; Sarah E. R. Bailey, 0000-0003-0020-4778.
} 
(a peripheral platelet level of $>400 \times 10^{9} / \mathrm{L}$ of blood) has been long recognised as a marker of poor prognosis in lung cancer patients (2). Recently thrombocytosis has been identified as having a particularly high predictive value for the identification of undiagnosed lung cancer (3), thus making it a promising biomarker in lung cancer diagnostics. However, lung cancer is heterogeneous, with multiple histological and genetic subtypes, and these subtypes each have distinct pathologies, treatment options, and prognoses. Histological lung cancer subtypes are broadly divided into non-small cell lung cancer (NSCLC), which represents $80-85 \%$ of all lung cancers, and small cell lung cancer (SCLC) which represents 15-20\%. NSCLC is further divided into adenocarcinoma (ADC), squamous cell carcinoma (SCC) and large cell carcinoma (LCC). There are several less common histological subtypes, including adenosquamous carcinoma, though distinguishing between these subtypes can be difficult. In addition to the diversity of the histological subtypes, lung cancers can also be distinguished by their genetic subtype. Most lung cancer genetic mutations are somatic, acquired through environmental factors such as tobacco smoking, and include driver mutations in genes such as EGFR, KRAS and TP53 and rearrangements in the $A L K$ gene. Currently, it is not known whether the platelet level differs across subtypes of lung cancer, and therefore whether thrombocytosis is a generic feature of lung cancer, or specific to some subtypes. The answer may facilitate improved lung cancer detection while also providing insights into the biological mechanism between lung cancer and thrombocytosis.

The aim of this systematic review and meta-analysis is to compare lung cancer patients' pre-treatment platelet counts across lung cancer subtypes to identify if thrombocytosis is more associated with a particular subtype(s). We present the following article in accordance with the PRISMA reporting checklist (available at http:// dx.doi.org/10.21037/tcr-20-3287).

\section{Methods}

\section{Protocol}

The execution of this systematic review and meta-analysis adhered to the study protocol which was published on PROSPERO on $13^{\text {th }}$ January 2020 [CRD42020162965 Available online: https://www.crd.york.ac.uk/prospero/ display_record.php?ID=CRD42020162965].

\section{Search strategy}

We identified relevant studies that reported the platelet count, either as an average or as the proportion of patients with thrombocytosis, by histological subtype of lung cancer. We searched PubMed and Embase on the $14^{\text {th }}$ January 2020 using search terms related to lung cancer and thrombocytosis or platelet count without a date limit (full search terms in Appendix 1). Inclusion criteria for the metaanalysis were: participants were diagnosed with a specific histological or genetic subtype of lung cancer; studies must have reported the mean platelet count or prevalence of thrombocytosis by subtype of lung cancer; reported mean platelet count or diagnosis of thrombocytosis before treatments such as surgery, chemotherapy or radiotherapy (as these can affect platelet counts); participants were aged 18 years or over. Two reviewers independently screened studies and conflicts were resolved by discussion. For studies that reported the same cohorts of participants, the study with the largest sample size was selected for the meta-analysis. Studies that reported median platelet count or did not use a thrombocytosis threshold of $400 \times 10^{9} / \mathrm{L}$ were excluded from the meta-analysis and were discussed narratively.

\section{Quality assessment}

Study quality was assessed using the Newcastle-Ottawa Scale (4) independently by three reviewers. Scoring was based on three domains: selection, comparability and exposure/outcome for a maximum of 9 points, based on the aims reported in the study rather than the aims of this review. The mean was taken of the three reviewers' scores and studies were judged as 'good', 'fair', or 'poor' quality based on the Newcastle-Ottawa Scale thresholds (4). As the main extracted data for this analysis were the histological diagnoses and average platelet count (or thrombocytosis proportions), rather than the outcome of the studies, studies rated as 'poor' were not excluded from the analyses; however, a sensitivity analysis investigated the potential influence of study quality on the findings.

\section{Data extraction}

The following data were extracted independently by two reviewers and cross-referenced for discrepancies: country of study, age of participants, sex ratio, smoking status, 
number of patients with each histological subtype, and average platelet count and/or proportion of patients with thrombocytosis. Study authors were contacted where data were missing or to confirm whether the inclusion criteria was met. Reminder emails were sent at two monthly intervals if no reply was received.

\section{Statistical analysis}

Analyses were carried out using STATA version 16. Random effects meta-analysis was used to pool the mean platelet count and the proportion of patients with thrombocytosis, using the meta set and metaprop commands, respectively. The restricted maximum likelihood estimation method was used to pool the mean platelet counts, and when pooling proportions the Freeman-Tukey double arcsine transformation was used to stabilise the variances. Findings were graphically displayed using forest plots. A subgroup analysis compared pooled mean platelet counts and the pooled proportions of patients with thrombocytosis across histological subtypes of lung cancer. The $\mathrm{I}^{2}$ statistic quantified heterogeneity. Sensitivity analyses explored potential sources of heterogeneity: first examining only advanced stage cancers, second removing the papers scoring 'poor' on the Newcastle-Ottawa Scale (4), and third removing studies that had a small sample size (less than 50 participants) for each histological subtype.

\section{Results}

\section{Data retrieval and study quality}

A total of 6,118 studies were returned from the search, including 1,352 duplicates. Abstracts of the remaining 4,766 studies were screened, resulting in 82 studies eligible for full-text screening. Thirty-eight studies met the criteria for the narrative synthesis with a total of twenty studies that fulfilled the inclusion criteria for meta-analysis (Figure 1). Eighteen studies were assessed as 'good' quality, five as 'fair', and fifteen as 'poor' according to the Newcastle-Ottawa Scale thresholds (5).

\section{Study characteristics}

There was a total of 2,974 patients across 20 different studies included in the meta-analyses (Tables 1,2). This consisted of 1,894 patients with ADC, 777 patients with SCC, 55 patients with LCC, and 248 patients with SCLC. Study countries included Bulgaria, Canada, China, Denmark, Greece, India, Japan, Korea, Poland, and Turkey. The ages ranged from 20 to 92 with a mean age of 58 years and median age of 63 years. Three-quarters (73.5\%) of participants were male. Sample size ranged from 17 to 347 with a median sample size of 61 . Twelve studies reported mean platelet counts and ten studies reported the proportion of patients with thrombocytosis (defined as a platelet level of over $400 \times 10^{9} / \mathrm{L}$ ); two studies reported both. Seven studies were assessed as 'good' quality, four as 'fair', and nine as 'poor'.

The search also returned an additional 18 studies with a further 6,917 lung cancer patients that did not fulfil the criteria for a meta-analysis, and are therefore discussed narratively. There was an inadequate number of studies per subtype for six of studies, seven studies defined thrombocytosis as a value other than $400 \times 10^{9} / \mathrm{L}$, and eleven reported median platelet count (some studies included more than one of these criteria). Eleven of these were assessed as 'good', one as 'fair', and six as 'poor' based on the Newcastle-Ottowa Scale (4).

\section{Meta-analysis of thrombocytosis proportions}

We pooled the proportions of patients with thrombocytosis (defined as $\left.400 \times 10^{9} / \mathrm{L}\right)(6-15)$. The overall pooled percentage of lung cancer patients with thrombocytosis was $27 \%$ (95\% CI: $17 \%$ to $37 \%$ ). By subtype, this proportion was $22 \%$ (95\% CI: $7 \%$ to $41 \%$ ) for ADC patients; $28 \%$ (95\% CI: $15 \%$ to $43 \%$ ) for SCC patients; $36 \%$ (95\% CI: $13 \%$ to $62 \%$ ) for LCC patients; and 30\% (95\% CI: $8 \%$ to $58 \%$ ) for SCLC patients (Figure 2). There was little evidence of differences in thrombocytosis prevalence between these subtypes $(\mathrm{P}=0.76)$. The heterogeneity $\left(\mathrm{I}^{2}\right.$ statistic) across subtypes was $97 \%$ for ADC, $92 \%$ for SCC, $61 \%$ for LCC, $86 \%$ for SCLC, and 93\% across all studies. This was not affected by any of the sensitivity analyses.

\section{Meta-analysis of mean platelet count}

Of the 12 studies that reported a mean platelet count $(11,12,16-25)$, the pooled mean across all histological subtypes of lung cancer was $289 \times 10^{9} / \mathrm{L}$ ( $95 \%$ CI: 268 to 311) (Figure 3). By subtype, the pooled mean platelet counts were $282 \times 10^{9} / \mathrm{L}$ (95\% CI: 259 to 306) for ADC; $297 \times 10^{9} / \mathrm{L}$ (95\% CI: 238 to 356 ) for SCC; $290 \times 10^{9} / \mathrm{L}$ 


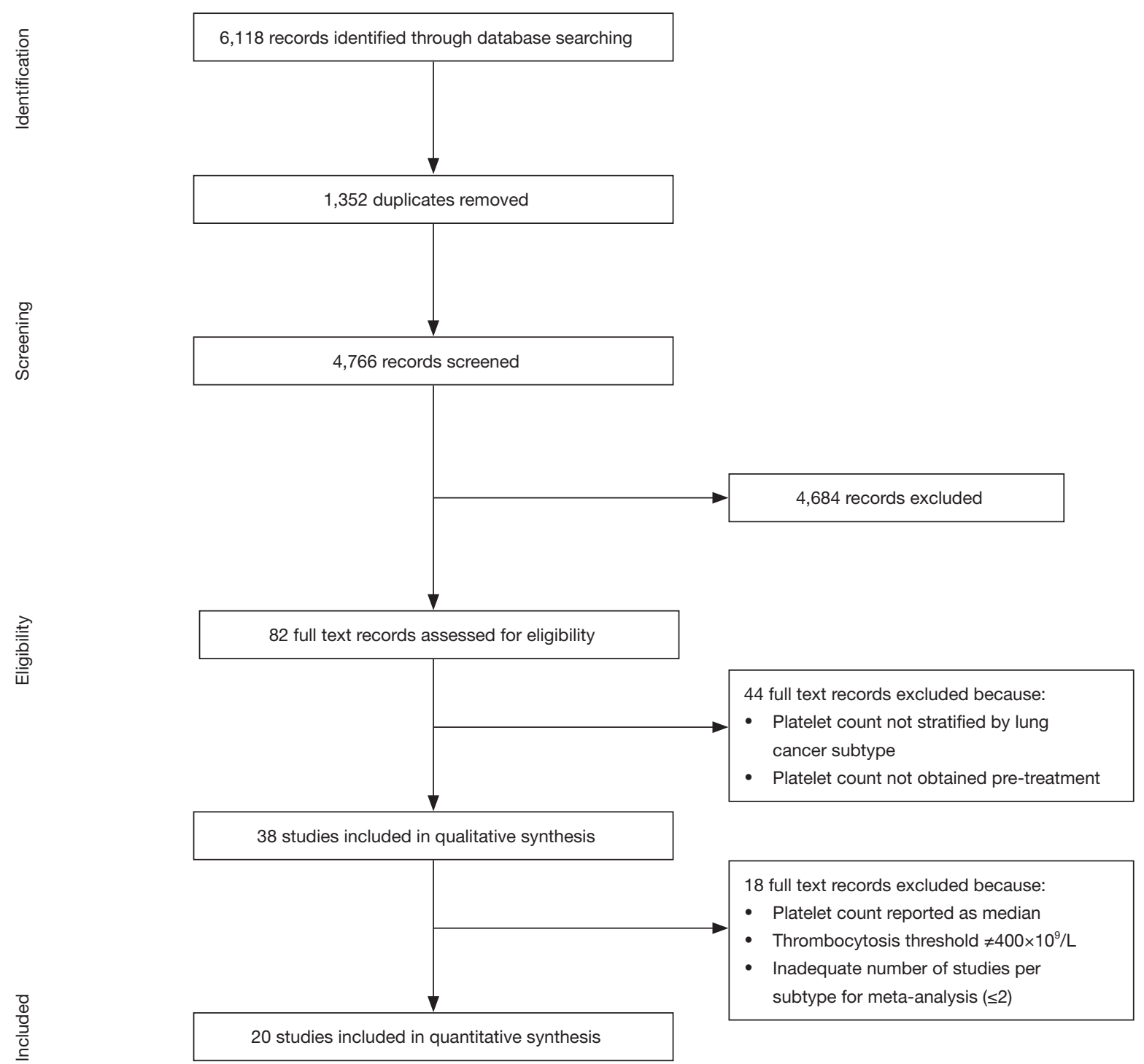

Figure 1 A flow diagram of the study selection process in accordance to PRISMA-P (5) guidelines.

(95\% CI: 176 to 404 ) for LCC; and $293 \times 10 \% / \mathrm{L}$ (95\% CI: 244 to 342 ) for SCLC. The $\mathrm{I}^{2}$ statistic for heterogeneity across studies was: $95 \%$ for ADC, $96 \%$ for SCC, $89 \%$ for LCC, $93 \%$ for SCLC, and 97\% overall. There was little evidence of differences in mean platelet count across the histological subtypes of lung cancer $(\mathrm{P}=0.96)$. None of the sensitivity analyses changed the main results; however, the analysis restricted to advanced stage cancers reduced heterogeneity for the ADC and SCC subgroups to $46 \%$ and $0 \%$, respectively. There were too few studies for LCC and SCLC to conduct a sensitivity analysis exclusively for advanced staged cancers.

\section{Median platelet counts and thrombocytosis proportions using different thresholds}

There was also little evidence of differences across lung cancer subtypes in 11 studies, inclusive of 4,887, lung cancer patients that reported median platelet count values (26-36), and seven studies of 2,795 lung cancer patients that used a different threshold to define thrombocytosis (28-30,36-39).

\section{Other subtypes of lung cancer}

Six studies, totalling 841 patients, were returned that examined platelet levels across other subtypes of lung 
Table 1 Characteristics of studies included in meta-analyses reporting thrombocytosis proportions (6-15)

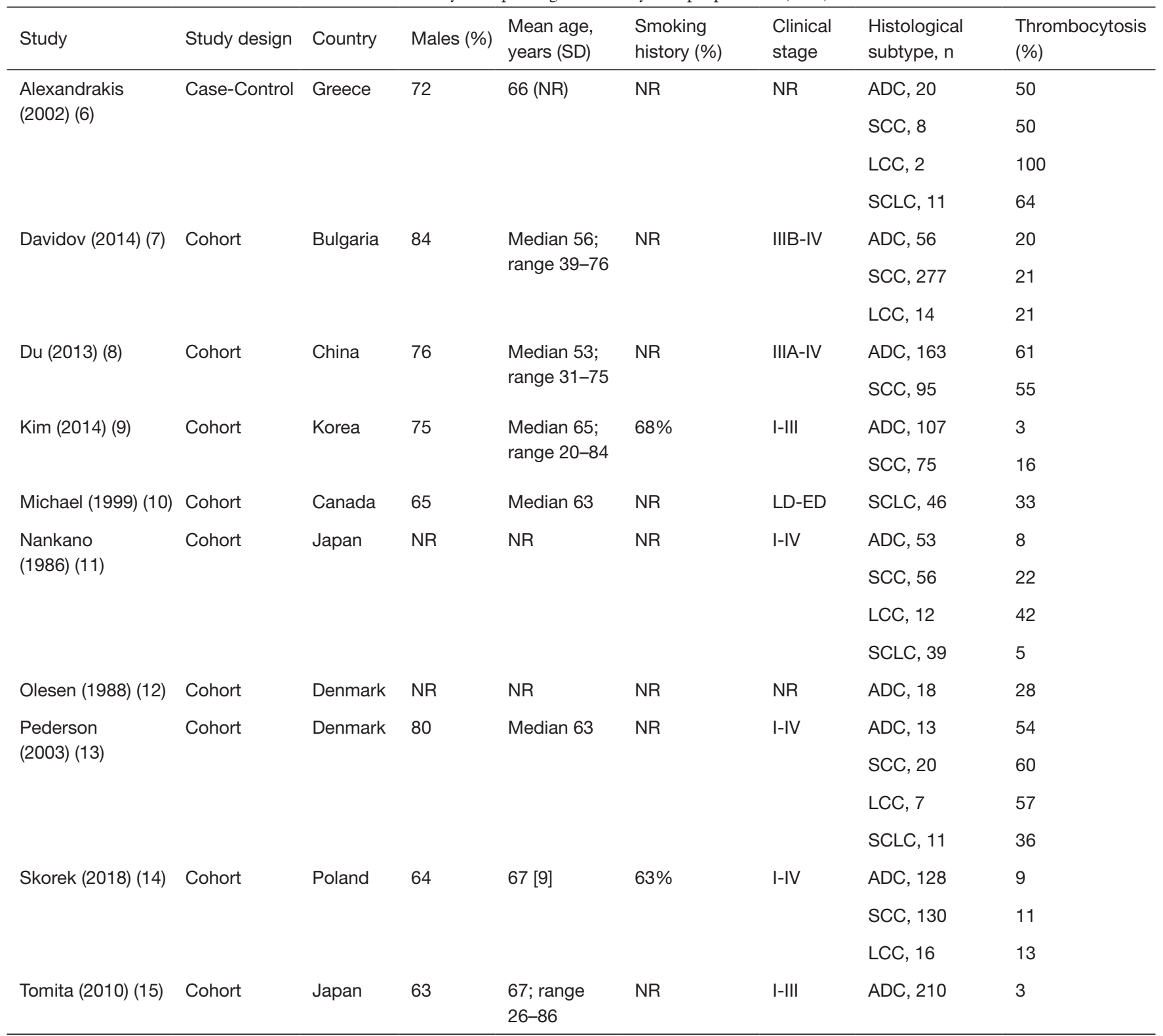

cancer (24,37-41).

\section{Adenosquamous carcinoma}

Two studies had available platelet counts or thrombocytosis proportions for patients with adenosquamous: Skorek and colleagues [2018] (14) reported 1/11 (9\%) patients with adenosquamous had thrombocytosis $\left(400 \times 10^{9} / \mathrm{L}\right.$ threshold $)$, and Wang and colleagues [2017] (40) reported a mean [SD] platelet count of 254 [72] across 134 patients.

\section{ALK rearrangements}

He and colleagues [2019] (41) studied 344 patients with ALK-positive lung cancer and stratified patients' platelet counts by percentage of ALK-positive tumour cells (15-49\% and $\geq 50 \%$ ), as detected by fluorescence in situ hybridisation. They reported $57 \%$ of patients with $15-49 \%$ of ALKpositive tumour cells and only $33 \%$ of patients with $\geq 50 \%$ ALK-positive tumour cells as having a platelet count of over $300 \times 10^{9} / \mathrm{L}$ blood. Yang and colleagues [2020] (42) reported 
Table 2 Characteristics of studies included in meta-analyses reporting mean platelet count $(11,12,16-25)$

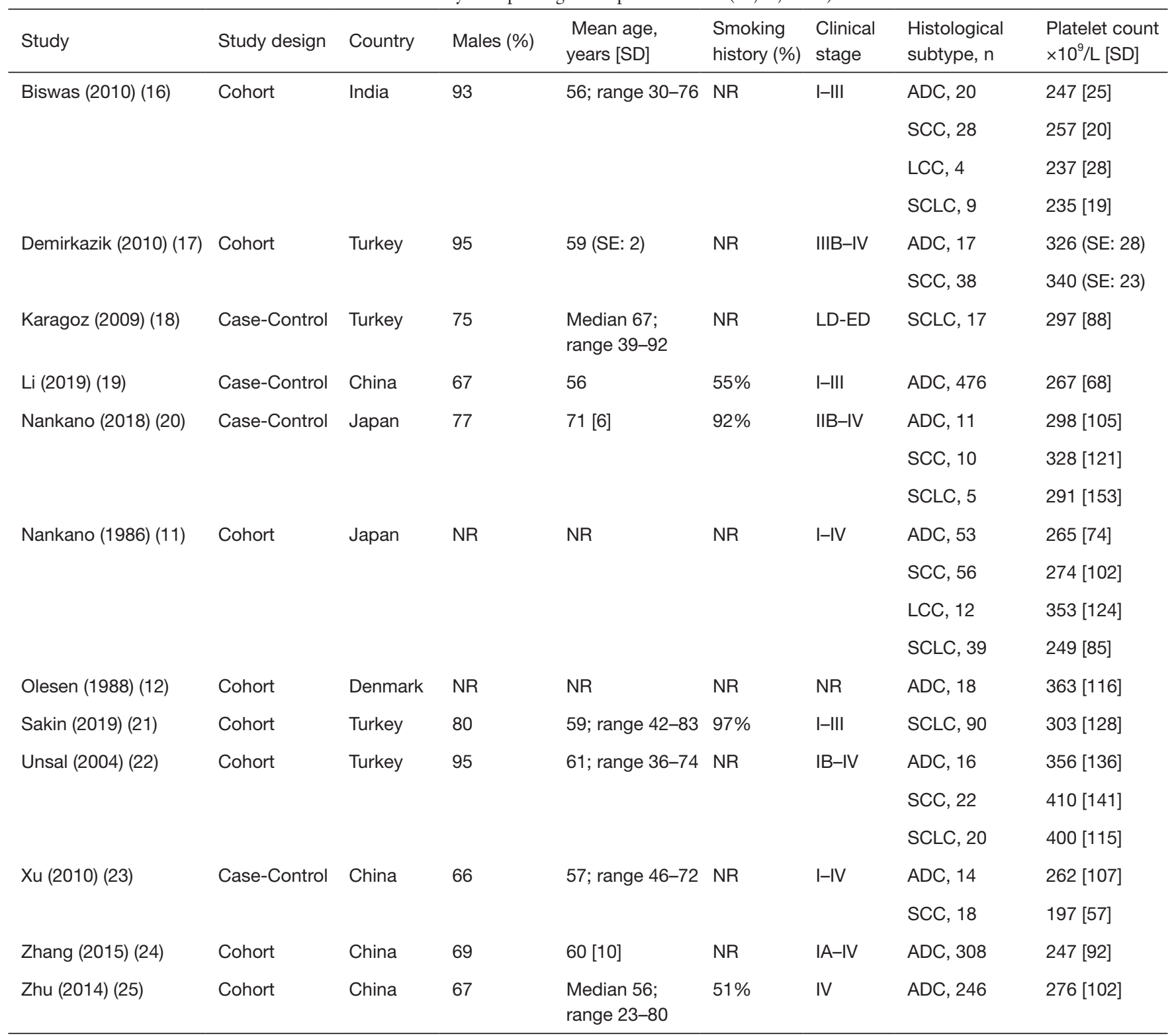

a median platelet count of 246 for 113 ALK-positive advanced NSCLC patients.

\section{EGFR mutations}

Two papers studied 112 (43) and 127 (44) advanced NSCLC patients, with either an EGFR exon 19 deletion or an EGFR exon 21 substitution mutation (L858R). They reported median platelet counts of 290 (43) and $232 \times 10^{9} / \mathrm{L}(44)$, respectively.

\section{Discussion}

This is the first systematic review to compare platelet count across different subtypes of lung cancer. Data from 2,974 patients across 20 independent studies (6-25) were synthesised and meta-analyses examined differences in mean platelet count and proportion of patients with thrombocytosis across ADC, SCC, LCC, and SCLC, finding no difference in mean platelet count or the 


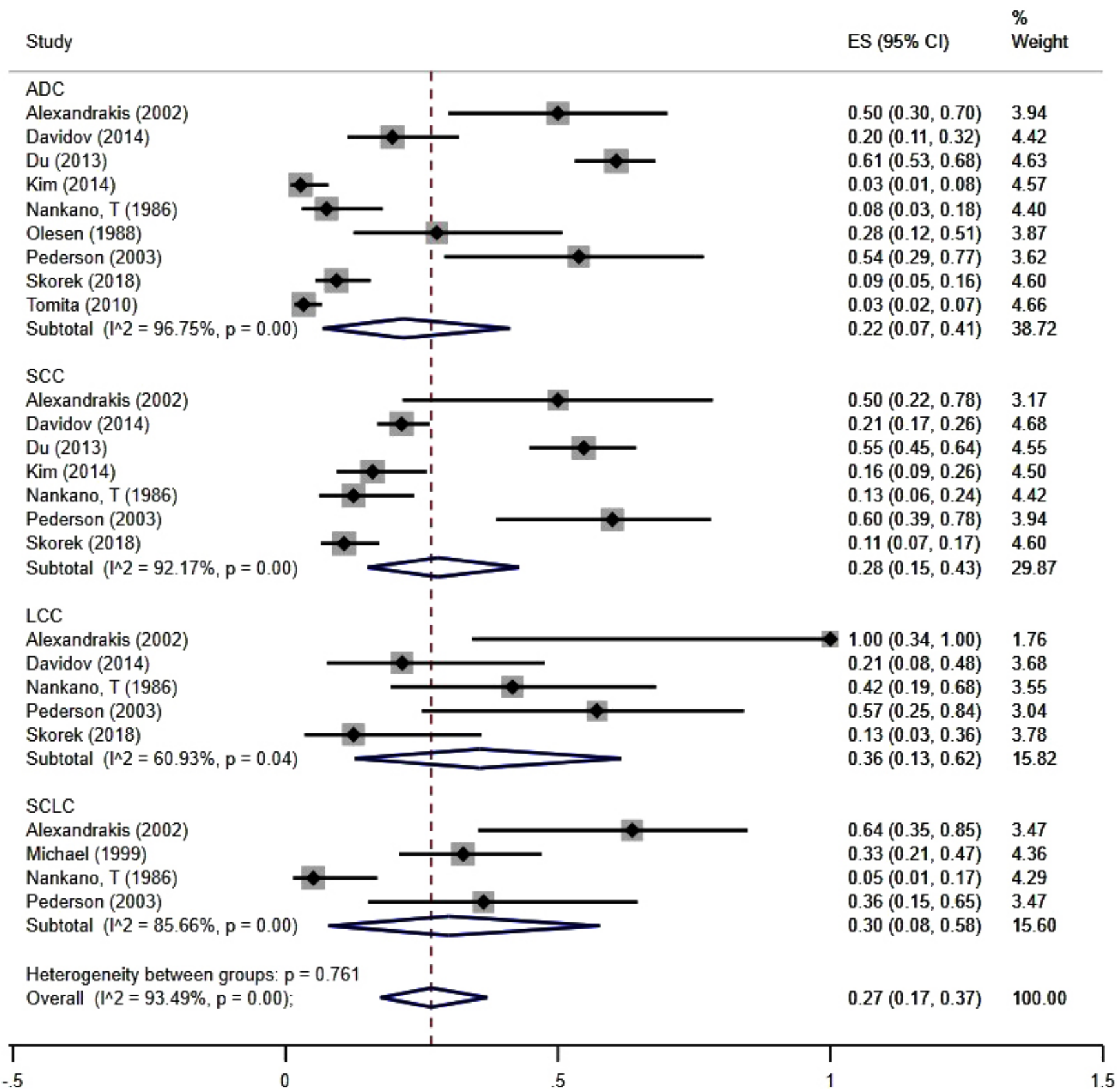

Figure 2 Random effects meta-analysis of studies that reported the proportion of patients with thrombocytosis (6-15). Bars, 95\% CI.

percentage with thrombocytosis across the four subtypes. Similar results were observed in a further 18 studies of 6,917 additional participants that reported median platelet counts (26-36) or proportion of patients with thrombocytosis using a lower threshold platelet count (28-30,36-39). There were too few papers on adenosquamous $(14,40), A L K$-positive $(41,42)$, and $E G F R$-positive $(43,44)$ lung cancers to perform a meta-analysis, although the available evidence suggested thrombocytosis was not a prominent feature of any of these subtypes. Our results indicate that the relationship between pre-treatment thrombocytosis and lung cancer is a generic one, rather than being restricted to one or more cancer subtypes. Diagnostically, finding that thrombocytosis was associated with all major subtypes is useful, as 


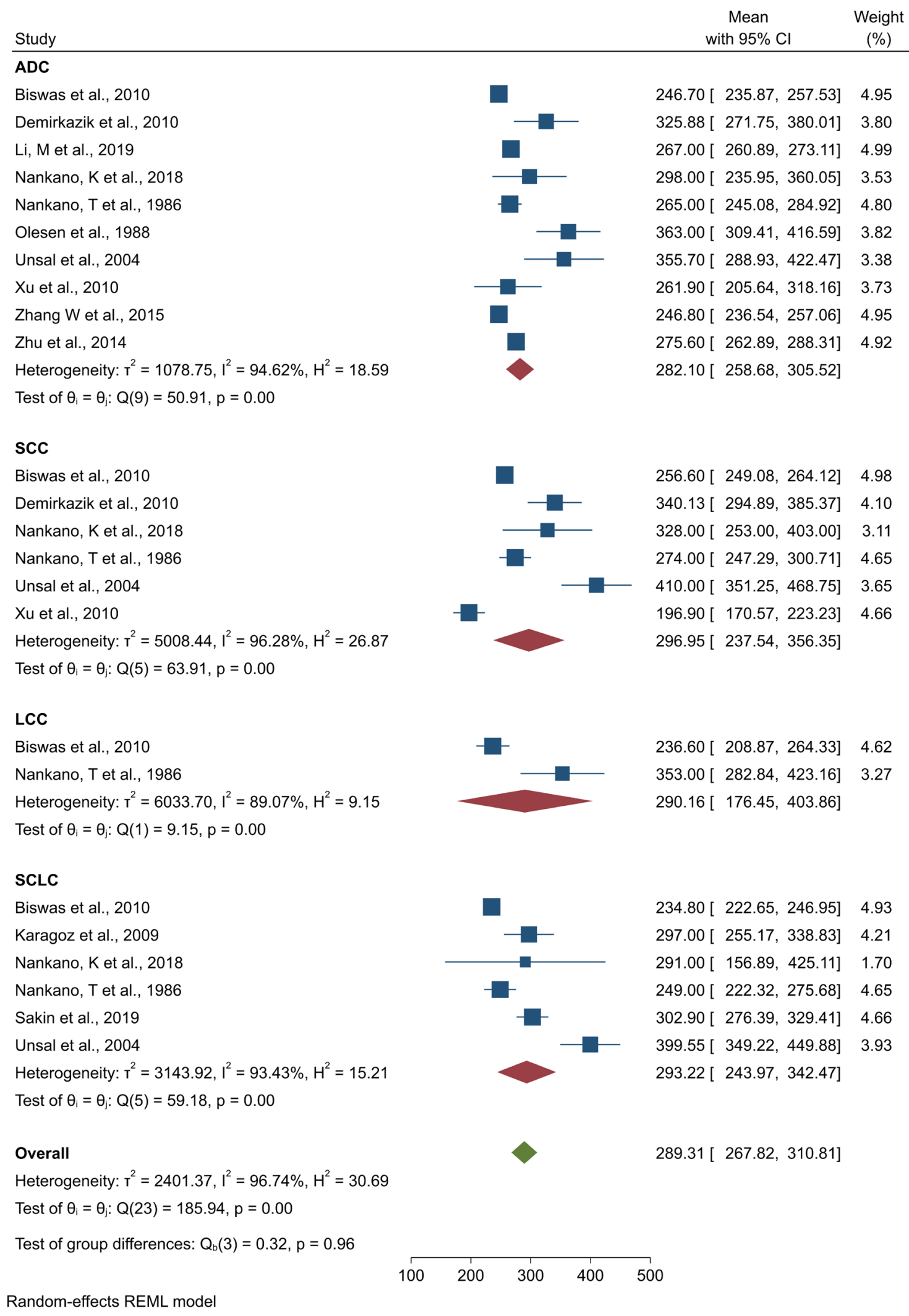

Figure 3 Random effects meta-analysis of studies that reported mean platelet count. (11,12,16-25) Bars, $95 \%$ CI. 
thrombocytosis is included in UK national guidance for lung cancer diagnosis, and we now know it is relevant for all major lung cancer subtypes.

\section{Strengths and limitations}

This systematic review collated data from a very large number of lung cancer patients to analyse platelet count and proportion of thrombocytosis. We were meticulous in studying only pre-treatment platelet values: lung cancer therapies such as surgery, chemotherapy, and radiotherapy can affect platelet count. While most studies were assessed as 'poor' based on the Newcastle-Ottawa Scale (4), all studies scored highly in the 'selection' domain. As the key data points extracted from each study were subtype diagnosis and platelet count, found in the patient characteristics rather than the results of the study, the 'selection' domain was the most important for the purpose of this review. Importantly, subtype diagnosis and platelet count were both obtained by robust, internationally recognised methods. The sensitivity analysis by study quality supported this. Furthermore, as this systematic review was concerned with patient characteristics (lung cancer subtype, platelet count) rather than the specific outcome of the included studies, any effect of publication bias should be small or absent.

There was a high level of heterogeneity throughout. To explore this, sensitivity analyses were conducted to analyse the effect of lung cancer stage, study quality and sample size. The sensitivity analysis that only included advanced lung stage cancers was the only one to reduce heterogeneity, perhaps because advanced stage cancers are more strongly associated with raised platelet count (2). Another source of heterogeneity may be attributed to smoking status, as smoking is not only a risk factor for lung cancer but also elevates the platelet count (45). Only a small number of studies reported smoking status but none differentiated smoking status in terms of platelet count and subtype: therefore, we were unable to analyse the effects of this.

\section{Comparison with the existing literature}

We identified $27 \%$ (95\% CI: $17 \%$ to $37 \%$ ) of lung cancer patients to have pre-treatment thrombocytosis; this is much higher than the $1.5-2.2 \%$ found in the general (healthy) population (3). This is in keeping with previous studies: Hamilton et al. [2005] (46) reported that $26 \%$ of lung cancer patients had thrombocytosis and Bailey et al. [2017] (3) found $23 \%$ of males with undiagnosed lung cancer present in primary care with thrombocytosis 12 months before their diagnosis. We also reported an overall mean platelet count of $289 \times 10^{9} / \mathrm{L}$ (95\% CI: 268 to 311 ) for lung cancer patients, again above the estimated mean platelet count of $258 \times 10^{9} / \mathrm{L}$ (SD: 62 ) for healthy individuals (47). As these figures did not vary between lung cancer subtypes, we propose there is a generic relationship between lung cancer and a raised platelet count across the major lung cancer subtypes. This may reflect the lungs being the site of $50 \%$ of platelet biogenesis (48), which could be stimulated by over-expression of cytokines and growth factors in the tumour microenvironment, irrespective of the tissue subtype. He et al., [2019] (41) reported a reduced proportion of thrombocytosis in patients with $\geq 50 \%$ ALK-positive tumour cells, compared to patients with $15-49 \%$ ALK-positive tumour cells, which may be suggestive of a negative association between $A L K$ translocations and thrombocytosis. As the only known study to report such a finding, it requires confirmation.

Platelets assist tumour metastasis in a number of ways. Tumour cells activate platelets upon dissemination, causing them to surround and protect the disseminated tumour cells from immune destruction and sheer stress to facilitate their haematological metastasis (49). Furthermore, platelet-derived growth factors promote endothelial permeability to expedite transendothelial migration for tumour extravasation in distant tissues and organs (50). Platelet-derived growth factors also stimulate tumour cell proliferation in order to establish and sustain secondary tumour growth at these distant sites. Of note, the platelet-derived growth factor pathway is regulated by the tumour suppressor p53, encoded by the TP53 gene, which is commonly mutated in lung cancer patients (51). Unfortunately, there is no existing literature regarding platelet count in lung cancer patients with TP53 mutations.

Whether thrombocytosis is due to the development of lung cancer or whether lung cancer development is enhanced by thrombocytosis is as yet unknown, and it may be truly bidirectional. Thrombocytosis can be a reactive condition that develops secondary to other disease, including lung cancer (52). However, a recent Mendelian randomisation analysis suggested a causal relationship between raised platelet count and increased lung cancer risk (53).

\section{Clinical implications}

These findings will be of interest to clinicians involved in 
diagnosing and estimating prognosis in lung cancer. Platelet count has long been used as a marker of prognosis in several cancer types; these results show that an elevated platelet count is no more strongly indicative of one lung cancer subtype over any other, and that lung cancer should be considered in a patient with an otherwise unexplained raised platelet count.

\section{Conclusions}

This systematic review and meta-analysis is the first to assess the relationship between platelet count and the subtypes of lung cancer. We report a $27 \%$ prevalence $(95 \%$ CI: $17 \%$ to $37 \%$ ) of thrombocytosis across lung cancer patients and a mean platelet count of $289 \times 10^{9} / \mathrm{L}(95 \%$ CI: 268 to 311). There was no evidence of differences in platelet levels across the major subtypes of lung cancer and therefore suggest that a thrombocytosis is likely to be a generic feature across all lung cancer subtypes.

\section{Acknowledgments}

Funding: This work was supported by the GW4 BIOMED DTP [grant MR/N0137941/1], awarded to the Universities of Bath, Bristol, Cardiff and Exeter from the Medical Research Council (MRC)/UKRI held by MB. This work was also supported by the CanTest Collaborative [funded by Cancer Research UK C8640/A23385] of which WH is a co-director, and from which SERB receives salary support. OCU was supported by the National Institute for Health Research (NIHR) Applied Research Collaboration SouthWest Peninsula (PenARC). The funding sources had no role in the study design, data collection, data analysis, data interpretation, writing of the report or in the decision to submit for publication.

\section{Footnote}

Reporting Checklist: The authors have completed the PRISMA reporting checklist. Available at http://dx.doi. org/10.21037/tcr-20-3287

Conflicts of Interest: All authors have completed the ICMJE uniform disclosure form (available at http://dx.doi. org/10.21037/tcr-20-3287). The authors have no conflicts of interest to declare.

Ethical Statement: The authors are accountable for all aspects of the work in ensuring that questions related to the accuracy or integrity of any part of the work are appropriately investigated and resolved.

Disclaimer: The views expressed in this publication are those of the authors and not necessarily those of the National Health Service, the NIHR or the Department of Health and Social Care.

Open Access Statement: This is an Open Access article distributed in accordance with the Creative Commons Attribution Licence (CC BY), which permits the noncommercial replication and distribution of the article with the strict proviso that no changes or edits are made and the original work is properly cited (including links to both the formal publication through the relevant DOI and the license). See: https://creativecommons.org/licenses/by-nc-nd/4.0/.

\section{References}

1. Office of National Statistics. Cancer survival in England - adults diagnosed 2019 [Available online: https:// www.ons.gov.uk/peoplepopulationandcommunity/ healthandsocialcare/conditionsanddiseases/datasets/cancer survivalratescancersurvivalinenglandadultsdiagnosed

2. Zhang X, Ran Y. Prognostic role of elevated platelet count in patients with lung cancer: a systematic review and metaanalysis. Int J Clin Exp Med 2015;8:5379-87.

3. Bailey SE, Ukoumunne OC, Shephard EA, et al. Clinical relevance of thrombocytosis in primary care: a prospective cohort study of cancer incidence using English electronic medical records and cancer registry data. Br J Gen Pract 2017;67:e405-e413.

4. Wells GA, Shea B, O'Connell D, et al. The NewcastleOttawa Scale (NOS) for assessing the quality of nonrandomised studies in meta-analyses 2013. Available online: http://www.ohri.ca/programs/clinical_ epidemiology/oxford.asp

5. Shamseer L, Moher D, Clarke M, et al. Preferred reporting items for systematic review and meta-analysis protocols (PRISMA-P) 2015: elaboration and explanation. BMJ 2015;350:g7647.

6. Alexandrakis MG, Passam FH, Perisinakis K, et al. Serum proinflammatory cytokines and its relationship to clinical parameters in lung cancer patients with reactive thrombocytosis. Respir Med 2002;96:553-8.

7. Davidov D. Thrombocytosis as prognostic factor for survival in patients with advanced non small cell lung 
cancer treated with first- line chemotherapy. Journal of IMAB 2014;20:560-4.

8. Du G, Yang Y, Zhang Y, et al. Thrombocytosis and immunohistochemical expression of connexin 43 at diagnosis predict survival in advanced non-small-cell lung cancer treated with cisplatin-based chemotherapy. Cancer Chemother Pharmacol 2013;71:893-904. Erratum in: Cancer Chemother Pharmacol 2013;72:275.

9. Kim M, Chang H, Yang HC, et al. Preoperative thrombocytosis is a significant unfavorable prognostic factor for patients with resectable non-small cell lung cancer. World J Surg Oncol 2014;12:37.

10. Michael M, Babic B, Khokha R, et al. Expression and prognostic significance of metalloproteinases and their tissue inhibitors in patients with small-cell lung cancer. J Clin Oncol 1999;17:1802-8.

11. Nakano T, Fujii J, Tamura S, et al. Thrombocytosis in patients with malignant pleural mesothelioma. Cancer 1986;58:1699-701.

12. Olesen LL, Thorshauge H. Thrombocytosis in patients with malignant pleural mesothelioma. Cancer 1988;62:1194-6.

13. Pedersen LM, Milman N. Diagnostic significance of platelet count and other blood analyses in patients with lung cancer. Oncol Rep 2003;10:213-6.

14. Skorek P, Stepien K, Fila M, et al. Preoperative thrombocytosis in surgically treated patients with non-small cell lung cancer. Pol Arch Intern Med 2018;128:512-7.

15. Tomita M, Shimizu T, Ayabe T, et al. Prognostic significance of the combined use of preoperative platelet count and serum carcinoembryonic antigen level in nonsmall-cell lung cancer. Gen Thorac Cardiovasc Surg 2010;58:573-6.

16. Biswas S, Sarkar S, Chakraborty J, et al. Occult micrometastasis to bone marrow in early lung cancer: a clinicopathologic study from West Bengal, India. Asian Pac J Cancer Prev 2010;11:747-51.

17. Demirkazik A, Asik M, Yalcin B, et al. The prognostic importance of leucocytosis, thrombocytosis and serum vascular endothelial growth factor (VEGF) level in inoperable non-small cell lung cancer (NSCLC): A matchpair analysis. UHOD 2010;20:14-9.

18. Karagoz B, Alacacioglu A, Bilgi O, et al. Platelet count and platelet distribution width increase in lung cancer patients. Anatolian Journal of Clinical Investigation 2009;3:32-4.

19. Li MM, Wang X, Yun ZY, et al. Platelet indices in nonsmall cell lung cancer patients with brain metastases.
Cancer Biomark 2019;24:515-9.

20. Nakano K, Sugiyama K, Satoh H, et al. Risk factors for disseminated intravascular coagulation in patients with lung cancer. Thorac Cancer 2018;9:931-8.

21. Sakin A, Yasar N, Arici S, et al. Effect of Pretreatment Platelet Parameters on Survival in Limited Disease Small Cell Lung Cancer. Asian Pac J Cancer Prev 2019;20:1879-85.

22. Unsal E, Atalay F, Atikcan S, et al. Prognostic significance of hemostatic parameters in patients with lung cancer. Respir Med 2004;98:93-8.

23. $\mathrm{Xu} \mathrm{C}, \mathrm{Fu} \mathrm{X}$. The changes of blood coagulation in surgical patients with lung cancer]. Zhongguo Fei Ai Za Zhi 2010;13:136-9.

24. Zhang W, Yu C, Huang B, et al. Correlation between bone metastasis and thrombocytosis in pulmonary adenocarcinoma patients. Oncol Lett 2015;9:762-8.

25. Zhu JF, Cai L, Zhang XW, et al. High plasma fibrinogen concentration and platelet count unfavorably impact survival in non-small cell lung cancer patients with brain metastases. Chin J Cancer 2014;33:96-104.

26. Bernhardt D, Aufderstrasse S, Konig L, et al. Impact of inflammatory markers on survival in patients with limited disease small-cell lung cancer undergoing chemoradiotherapy. Cancer Manag Res 2018;10:6563-9.

27. de Campos E, Radford J, Steward W, et al. Clinical and in vitro effects of recombinant human erythropoietin in patients receiving intensive chemotherapy for small-cell lung cancer. J Clin Oncol 1995;13:1623-31.

28. Fang L, Sheng H, Wan D, et al. Prognostic role of multidrug resistance-associated protein 1 expression and platelet count in operable non-small cell lung cancer. Oncol Lett 2018;16:1123-32.

29. Hong X, Xu Q, Yang Z, et al. The value of prognostic factors in Chinese patients with small cell lung cancer: A retrospective study of 999 patients. Clin Respir J 2018;12:433-47.

30. Ji Y, Sheng L, Du X, et al. Elevated platelet count is a strong predictor of poor prognosis in stage I non-small cell lung cancer patients. Platelets 2015;26:138-42.

31. Li SQ, Jiang YH, Lin J et al. Albumin-to-fibrinogen ratio as a promising biomarker to predict clinical outcome of non-small cell lung cancer individuals. Cancer Med 2018;7:1221-31.

32. Li Y, Wei S, Wang J, et al. Analysis of the factors associated with abnormal coagulation and prognosis in patients with non-small cell lung cancer]. Zhongguo Fei Ai Za Zhi 2014;17:789-96. 
33. Phan TT, Nguyen ATT, Van Nguyen AN, et al. Neutrophil to lymphocyte with monocyte to lymphocyte ratio and white blood cell count in prediction of lung cancer. Australas Med J 2018;11:231-6.

34. Shi L, Li Y, Yu T, et al. Predictable Resistance and Overall Survival of Gemcitabine/Cisplatin by Platelet Activation Index in Non-Small Cell Lung Cancer. Med Sci Monit 2018;24:8655-68.

35. Suzuki R, Wei X, Allen PK, et al. Hematologic variables associated with brain failure in patients with small-cell lung cancer. Radiother Oncol 2018;128:505-12.

36. Yu D, Liu B, Zhang L, et al. Platelet count predicts prognosis in operable non-small cell lung cancer. Exp Ther Med 2013;5:1351-4.

37. Abraham VT, Radhakrishna BS, Abdulwahab MO. A study of paraneoplastic syndrome patterns in patients with bronchogenic carcinoma. Bahrain Medical Bulletin 2005;27:172-4.

38. Demirci NY, Turay UY, Yilmaz A, et al. Vascular events in lung cancer. Asian Pac J Cancer Prev 2011;12:2685-7.

39. Gonzalez Barcala FJ, Garcia Prim JM, Moldes Rodriguez $\mathrm{M}$, et al. Platelet count: association with prognosis in lung cancer. Med Oncol 2010;27:357-62.

40. Wang YQ, Zhi QJ, Wang XY, et al. Prognostic value of combined platelet, fibrinogen, neutrophil to lymphocyte ratio and platelet to lymphocyte ratio in patients with lung adenosquamous cancer. Oncol Lett 2017;14:4331-8.

41. He Y, Gong R, Sun LY, et al. The Percentage of Anaplastic Lymphoma Kinase-Positive Tumor Cells Has Clinical Implications for Patients with Non-Small Cell Lung Cancer. Genet Test Mol Biomarkers 2019;23:589-97.

42. Yang $Y, X u$ H, Yang G, et al. The value of blood biomarkers of progression and prognosis in ALK-positive patients with non-small cell lung cancer treated with crizotinib. Asia Pac J Clin Oncol 2020;16:63-9.

43. Phan TT, Ho TT, Nguyen HT, et al. The prognostic impact of neutrophil to lymphocyte ratio in advanced non- small cell lung cancer patients treated with EGFR TKI. Int J Gen Med 2018;11:423-30.

44. Zhang Y, Feng YC, Zhu HG, et al. The peripheral blood neutrophil-to-lymphocyte ratio is a prognostic predictor for survival of EGFR-mutant nonsmall cell lung cancer patients treated with EGFR-TKIs. Medicine (Baltimore) 2018;97:e11648.

45. Erikssen J, Hellem A, Stormorken H. Chronic effect of smoking on platelet count and "platelet adhesiveness" in presumably healthy middle-aged men. Thromb Haemost 1977;38:606-11.

46. Hamilton W, Peters TJ, Round A, et al. What are the clinical features of lung cancer before the diagnosis is made? A population based case-control study. Thorax 2005;60:1059-65.

47. Demirin H, Ozhan H, Ucgun T, et al. Normal range of mean platelet volume in healthy subjects: Insight from a large epidemiologic study. Thromb Res 2011;128:358-60.

48. Lefrançais E, Ortiz-Muñoz G, Caudrillier A, et al. The lung is a site of platelet biogenesis and a reservoir for haematopoietic progenitors. Nature 2017;544:105-9.

49. Gay LJ, Felding-Hebermann B. Contribution of platelets to tumour metastasis. Nat Rev Cancer 2011;11:123-34.

50. Schlesinger $M$. Role of platelets and platelet receptors in cancer metastasis. J Hematol Oncol 2018;11:125.

51. Farooqi AA, Siddik ZH. Platelet-derived growth factor (PDGF) signalling in cancer: rapidly emerging signalling landscape. Cell Biochem Funct 2015;33:257-65.

52. Werynska B, Ramlau R, Podolak-Dawidziak M, et al. Serum thrombopoietin levels in patients with reactive thrombocytosis due to lung cancer and in patients with essential thrombocythemia. Neoplasma 2003;50:447-51.

53. Zhu Y, Wei Y, Zhang R, et al. Elevated Platelet Count Appears to Be Causally Associated with Increased Risk of Lung Cancer: A Mendelian Randomization Analysis. Cancer Epidemiol Biomarkers Prev 2019;28:935-42.
Cite this article as: Barlow M, Hamilton W, Ukoumunne OC, Bailey SER. The association between thrombocytosis and subtype of lung cancer: a systematic review and meta-analysis. Transl Cancer Res 2021;10(3):1249-1260. doi: 10.21037/tcr20-3287 


\section{Supplementary}

Appendix 1 Search strategy

\section{Medline (Ovid) and EMBASE}

1. $\quad$ exp Lung Neoplasms/ (MEsH term)

2. lung*.ti,ab.

3. pulmonary*.ti,ab.

4. respirat*.ti,ab.

5. bronch*.ti,ab.

6. 2 or 3 or 4 or 5

7. cancer*.ti,ab.

8. neoplasm*.ti,ab.

9. tumo? $\mathrm{r}^{*} . \mathrm{ti}, \mathrm{ab}$.

10. malign*.ti,ab.

11. carcinoma*ti,ab.

12. adenocarcinoma*.ti,ab.

13. 7 or 8 or 9 or 10 or 11 or 12

14. 6 adj3 13

15. nsclc*.ti,ab.

16. sclc $^{*} \cdot \mathrm{ti}, \mathrm{ab}$.

17. 15 or 16

18. 1 or 14 or 17

19. exp Blood Platelets/ (MEsH term)

20. exp Platelet Count/ (MEsH term)

21. exp Thrombocytosis/ (MEsH term)

22. platelet*.ti,ab.

23. platelet count*.ti,ab.

24. thrombocyte count* ti,ab.

25. thrombocytosis*.ti,ab.

26. megakaryocyt ${ }^{\star} . t \mathrm{ti}, \mathrm{ab}$.

27. 19 or 20 or 21 or 22 or 23 or 24 or 25 or 26

28. 18 and 27 